\title{
Die mindel-riß-interglaziale Landoberfläche am Westrand des Salzachvorlandgletschers
}

\author{
Von Edith E bers, München
}

Von den spärlichen Überresten dieser Landoberfläche im quartären Schichtensystem des nördlichen Alpenvorlandes sind die am Westrande des Salzachvorlandgletschers noch erhaltenen auf eine bisher noch nicht beobachtete Weise stratigraphisch eingeordnet.

Am Außenrande der von alpinen Vorlandgletschern erreichten Räume gibt es Gebiete, wo die Erosionswirkung der Vereisungen nur mehr gering und auch ihre Akkumulation nicht mehr so mächtig war.

Solche Stellen sind sehr günstig für quartärstratigraphische Beobachtungen im Alpenvorland. Man kann hier manchmal sehr schön und regional die Auflagerungs- oder Grenzfläche sowohl der würm- über die riß-eiszeitlichen, wie der riß- über die mindel-eiszeitlichen Bildungen erkennen. In diesen Randzonen sind die Profile meist vollständiger, weil keine allzu großen Erosionslücken miteinzurechnen sind. Immer wieder tauchen zwar Reste der alten Landoberfläche auf. Große durchgehende Verwitterungsrinden finden sich aber nur sehr selten. Ein stratigraphisch eingebauter Verwitterungshorizont der mindel-riß-interglazialen Landoberfläche ist beispielsweise bekannt aus dem „Kalten Tal“ (zwischen Schongau und Kaufbeuren) im Lechgletscher-Gebiet oder von Hauerz (bei Biberach) und Rottum im östlichen Rheingletschergebiet. Es handelt sich dabei immer um rotgetönte Vermitterungsmassen mindel-eiszeitlicher Bildungen.

Am Westrande des Salzachgletschers nun, da wo die mindel- und riß-eiszeitlichen Ablagerungen vom jungen Tale der $\mathrm{Alz}$ angeschnitten und aufgeschlossen werden, sind innerhalb der quartären Schichtenfolge Fetzen dieser Landoberfläche in Gestalt von Fragmenten der Verwitterungsschicht, von einstiger Lößdecke und Bodenbildungen zu finden.

Solche Überreste sind von Bedeutung für die sichere Gliederung des präalpinen Quartärs. Sie haben den Vorzug der unbedingten Eindeutigkeit. Die übrigen, schon seit PENCK verwandten Gliederungselemente, besonders die verschieden hohen Tertiärsockel, haben Enttäuschungen gebracht. Ihr Wert ist ein relativer, da sie nur ein Ausdruck verschiedener Gefällsverhältnisse sind, bei denen andere Faktoren als klimatische, insbesondere tektonische, mitspielen können. Eindeutige Interglaziale, wie sie etwa das Eem-Meer oder die HolsteinSee im norddeutschen Diluvium bieten, sind im nordalpinen Quartär nicht vorhanden. Die spärlichen Schieferkohlen sind noch nicht genügend durchuntersucht, als daß man mit ihrer Hilfe immer die Frage „Interglazial oder Interstadial?" beantworten könnte.

So sind und bleiben, nach Ansicht der Verfasserin, durchgehende Verwitterungshorizonte oder an deren Stelle regional auftretende Fetzenhorizonte von solchen das sicherste Gliederungselement.

Am Westrande des rißeiszeitlichen Salzachgletschers, in der Gegend von Feichtn (bei Wiesmühl a. d. Alz), Burghausen usw. stellen sich der morphologischen Analyse große Schwierigkeiten entgegen. Das „Altmoränengebiet“ ist zwar einer direkten Einwirkung des Würmeises nicht mehr ausgesetzt gewesen, abgesehen von den Einschnitten zweier würmeiszeitlicher Schmelzwassertäler, die es durchziehen: das „Schnitzinger Eisrandtal“ und das Brunntal. Es nivellieren aber Terrassenkreuzungen das Relief. Ebenerdig und unmerklich kann man bei Feichtn von der Deckenschotterplatte auf die Hochterrassenfläche und von dieser, bei Burghausen, auf die Niederterrassenfläche hinüberwechseln. Trennende 
Steilränder fehlen also. Penck \& Brückner haben dieses Gebiet als das der „äußeren Moräne“ bezeichnet. Tatsächlich wird es aber hauptsächilich von einer gewaltigen, peripher zur Vereisungszone angeordneten Hochterrasse eingenommen. Sie geht aus einem Altmoränenkranz hervor, welcher, aus mehreren Moränenwallzügen bestehend, dem proximal liegenden, zweiten, jüngeren und hauptsächlichsten Hochstande des Rißeises zugehörte. Distalwärts umhüllt diese Hochterrasse ältere Stauchmoränen; sie brandet an an den mit Mindelmoränen verknüpften und mit Rißmoränen bedeckten Deckenschotterstrang des Alztales Stein-Feichtn-Wald a. d. Alz-Margarethenberg-Höresham, und an die Mindelmoränen tragenden Deckenschotterinseln des Eschelberges und des Hechenberges. Ja sie überlappt transgredierend den Deckenschotterzug des Alztales teilweise selbst wieder zwischen Feichtn und Wald a. d. Alz mit einer Terrassenkreuzung, um, über den jüngeren Einschnitt des Brunntales hinweg, sich mit den Hochterrassen des Inngletschers zu vereinigen. Zwischen Höresham und dem Eschelberg bei Burgkirchen streicht die Hochterrasse ebenfalls, später von der Mündung des „Schnitzinger Eisrandtales“, dem Alztal und der Niederterrasse des Öttinger Forstes unterbrochen, zur Inngletscher-Hochterrasse hinüber.

Das Gebiet der Terrassenkreuzung ist es, wo die wirklich instruktiven Aufschlüsse zu holen sind. Die von der Seitenerosion der Rißschmelzwässer überwältigte Deckenschotteroberkante war hier glücklicherweise ehedem von ganzen Galerien mindel-riß-interglazialer geologischer Orgeln durchsetzt. Der östliche Rand des Alztales bei Tacherting zeigt ihre Überreste am Wege nach Mankham und auch südlich davon in alten Steinbrüchen und am Gehänge in großer Zahl und dichter Anordnung. Die Riß-Schmelzwässer haben sie, haarscharf wie mit dem Messer, abgeschnitten und nur die untersten, 1-2 m langen Orgelspitzen übriggelassen. Der Deckenschotter war also schon verfestigt und bereits wieder tiefgründig verwittert, als die neue Vereisung ihre Sedimente ablagerte. Dieser Horizont markiert die Diskordanz zwischen Mindel und Riß sehr deutlich. Selbstverständlich sind Orgeln nicht überall vorhanden, und auch im Terrassenkreuzungsgebiet ist es dann viel schwieriger, auf diese Diskordanzfläche die Hand zu legen. Die Riß- und Mindelablagerungen können sich sehr ähnlich sein, sogar hinsichtlich ihres Kristallingehaltes, wenn auch im allgemeinen das jüngere Riß sehr viel reicher an kristallinem Erratikum wird. Die Diskordanz hebt sich oft kaum ab.

Umso nützlicher wird nun hier jenes bei der Gliederung des alpinen Pleistozäns bisher noch nicht verwendete Phänomen: das Auftreten von Horizonten mit Schollen aus Verwitterungs- und Lößmaterial, die 1-2 m oberhalb der Diskordanz im jüngeren Sediment liegen. Sie sind meist $1-2 \mathrm{~m}$ lang oder auch kürzer, $1 / 2 \mathrm{~m}$ und mehr hoch und von mehr oder minder ebenen Flächen begrenzt. Meist stehen sie auf dem Kopf, woraus sich ableiten läßt, daß sie, wohl in gefrorenem Zustand, von einer ehemaligen Landoberfläche abgestürzt sind und eingebettet wurden. Die Schmelzwässer, die die Orgeln köpften, werden dieselben sein, die bei der Seitenerosion Flußufer unterwühlt und dabei die Schollen zum Absturz gebracht haben.

Meist bestehen die Schollen aus den ehemaligen Deckschichten der Deckenschotternagelfluh, entkalktem, rötlich gefärbten Verwitterungslehm, der manchmal noch im Verband mit jetzt darunter liegendem Lößlehm bleibt. Es gibt auch Schollen nur aus rötlich verlehmten Löß oder auch aus nur entkalktem gelben Löß. Hervorzuheben ist, daß dieser Löß, da bereits verlehmt, nicht wohl aus der Zeit des Hochstandes der heranrückenden Riß-Vereisung stammen kann, wie das der heutigen Lößtheorie entspräche. 
Mehrere solcher Schollen, die, von der Landoberfläche abstürzend, in das jüngere Sediment gerieten, liegen meist im selben Niveau wie an einem Bande aufgereiht nebeneinander, und bei fortschreitender Kiesgrubenarbeit tauchen immer wieder neue auf, während die alten verschwinden. Sie liegen meist in einem, nur einige Meter mächtigen Paket relativ lockeren Riß-Schotters, den man als eine Vorstoßbildung ansehen wird. Es kann wohl kein Zweifel bestehen, daß es sich dabei um Überreste der mindel-riß-interglazialen Landoberfläche handèlt. Solche Schollen dürfen natürlich nicht verwechselt werden mit ähnlich aussehenden Vorkommnissen anderer Herkunft, etwa verwitterten Geschiebemergelbänken, eingestauchten Schollen in Stauchmoränen, Grundwassermarken usw. Letztere Verwechslung wäre besonders im östlichen Rheingletschergebiet möglich, wo in den glazialen Sedimenten die zahlreichen hochliegenden Grundwassermarken besonders auffallen. Es kommt auch vor, daß auf der alten Landoberfläche das Verwitterungsmaterial aufgearbeitet wurde und in die unteren Riß-Schichten mit einging. Der starke Lehmgehalt dieser ist bekannt. Man findet in ihnen aber auch Schnüre von Lößgeröllen und - selbst im frischen Sediment - stark angewittertes Kristallinerratikum, das aus ältereiszeitlichen Ablagerungen stammen dürfte.

Das regionale Auftreten eines solchen Fetzenhorizontes in den basalen Schichten der Hochterrasse beweist, daß Seitenerosion und Akkumulation zur Zeit der beginnenden Bildung der Hochterrasse in diesem Gebiet unbedingt gleichzeitig vor sich gingen. Diese Beobachtung steht im Gegensatz zu den Ausführungen J. Schaefer's in „Die diluviale Erosion und Akkumulation, 1950.

Am Westrande des Salzachvorlandgletschers wurde bisher in drei Kiesgruben (bei Mankham gegenüber Tacherting, bei Amsham an der Straße Wiesmühl a. d. Alz-Feichtn und bei Schmidstadt an der Bahnlinie Garching-Kirchweidach) der Fetzenhorizont der mindel-riß-interglazialen Landoberfläche festgestellt. Sie liegt dort um $485 \mathrm{~m}$ ü. M. herum, nur etwa $20 \mathrm{~m}$ über dem heutigen Alzspiegel.

Während eine dicke Verwitterungsschicht von der riß-würm-interglazialen Landoberfläche, die immer wieder in einer Kiesgrube in der Niederterrasse bei Palling im bayrischen Salzachgletscherflügel aufgedeckt wird, nur wenig rötliche Braunfärbung aufweist, haben die Mindel-Riß-Interglazialschollen durch weg eine auffallend rote Tönung. Diese erinnert an den „ferretto“ vom Süd-

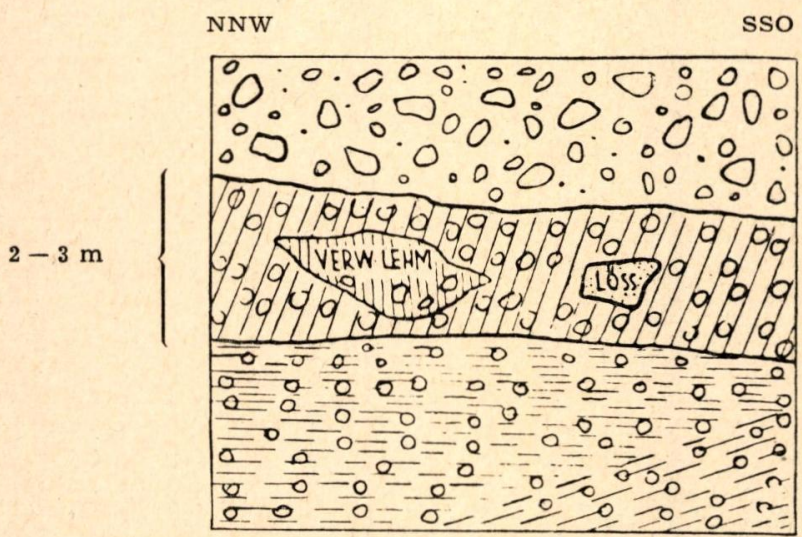

Abb. 1. Verstürzte Schollen von der mindelriß-interglazialen Landoberfläche, in RißVorstoß-Schotter eingebettet. Hangend Rißmoräne, liegend Mindelschotter. Schmidtstadt, Kiesgrube an der Bahnlinie Garching-Kirchweidach der "Tauernbahn“. Vergleiche Topograph. Atl. v. Bayern, 1: 25000 Bl. 700 (Engelsberg) und 722 (Trostberg). 
rande der Alpen, wie er mindel-eiszeitliche Moränen und Schotter dort bedeckt. Sie erinnert auch an die $3 \mathrm{~m}$ mächtige Rotlehmschicht im Lößprofil des LaerBerges bei Wien, welche auf ein warmes, mäßig feuchtes Klima im selben Zeitraume hinweist (siehe KüMEL, Führer für die Quartärexcursionen in Österreich, I. Teil, Wien 1936).

Unter den an sich wenig zahlreichen Interglazialablagerungen aus der Mindel-Riß-Interglazialzeit des Alpengebietes ragt die Höttinger Breccie hervor mit ihrem pontischen Rhododendron und Buxus sempervirens, die auf eine um $2^{\circ}$ höhere mittlere Jahrestemperatur und eine um $400 \mathrm{~m}$ höhere Lage der Schneegrenze als heute hinweisen. Damit zeigt sich ein wärmeres Klima an. Rotfärbung ist charakteristisch für subtropische Böden, eine Rötlichfärbung der Verwitterungsschichten wird man ebenfalls einem wärmeren Klima zuschreiben dürfen. Der geringere Humusgehalt wird als eine Folge höherer Wintertemperaturen und vorherrschender Winterregen angesehen. Die Rottönung ist auf Anreicherung kolloidaler Eisenoxyde zurückzuführen.

Diese der Höttinger Breccie gleichaltrigen Schollen rötlich getönter Verwitterungsböden aus dem Fetzenhorizont an der Basis der Hochterrasse oder der Rißmoränen wäre einer bodenkundlichen und chemischen Untersuchung wert. Sie könnten, in Verbindung mit anderen gleichzeitigen Bildungen gebracht, vielleicht Rückschlüsse auf das mindel-riß-interglaziale Klima erlauben.

$\mathrm{Z}$ us a m m enfas ung.

Am Westrande des ehemaligen Salzachvorlandgletschers, im Gebiete der ältereiszeitlichen Terrassenkreuzungen, ist der Deckenschotter der Mindeleiszeit stellenweise von Rißablagerungen zugedeckt worden, nachdem er eine leichte Erosion seiner Oberfläche erfahren hatte. Diese war zuvor von Löß und Lößlehm und rot getönten Verwitterungsschichten verhüllt und von Geologischen Orgeln durchsetzt gewesen. Der Abtrag ergriff nur die obersten Meter des Sediments, sodaß die Diskordanzfläche noch im B-Horizont steckenblieb. Auf dieser Diskordanzfläche haben wir Überreste der mindel-riß-interglazialen Landoberfläche aus jenem Zeitabschnitt, wo sie von den rißeiszeitlichen Schmelzwässern angegriffen und überschottert wurde. Die Diskordanzfläche ist, nicht überall kenntlich, in das quartäre Schichtensystem des Gebietes eingebaut. Wo sie aber durch die oben beschriebenen Schollen aus interglazialer Verwitterungsrinde, Lößlehm oder Löß, auf deren Entstehungsart und Alter die 1-2 m tiefer liegende erosive Schnittfläche der Deckenschotterorgeln Schlußfolgerungen erlaubt, besetzt ist, läßt sie sich, auf weitere Strecken hin und bis auf einige Meter genau, höhenmäßig gut festlegen.

Ms. eingeg.: 22. 6. 1951. 